\title{
Continuous Lumbar Plexus Catheter Removal in Postoperative Total Hip Replacement Patients Receiving Warfarin Thromboprophylaxis: A Retrospective Analysis
}

\author{
Joseph Marino' ${ }^{\text {, Jay Curtin }}{ }^{2}$, Carol Patrick ${ }^{3}$, Kara Theal ${ }^{4}$, Cristina Sison ${ }^{5}$, George Koutsouras ${ }^{5}$, \\ Scott Alpert ${ }^{6}$, W. Healy III $^{6}$, Oonagh Dowling ${ }^{7}$, Renee Pekmezaris ${ }^{7}$, J. E. Chelly ${ }^{8}$ \\ ${ }^{1}$ Department of Anesthesiology, Franklin Hospital Medical Center, Valley Stream, USA; ${ }^{2}$ Department of Anesthesiology, Huntington \\ Hospital, Huntington, USA; ${ }_{3}^{3}$ Department of Cardiology, Huntington Hospital, Huntington, USA; ${ }^{4}$ Department of Information Ser- \\ vices, Huntington Hospital, Huntington, USA; ${ }^{5}$ Biostatistics Unit, The Feinstein Institute for Medical Research, Manhasset, USA; \\ ${ }^{6}$ Department of Orthopedics, Huntington Hospital, Huntington, USA; ${ }^{7}$ Department of Population Health, North Shore LIJ Health \\ System, Great Neck, USA; ${ }^{8}$ Department of Orthopedic Anesthesia and Acute Interventional Pain, University of Pittsburgh School of \\ Medicine, Pittsburgh, USA.
}

Email: jmarino7@nshs.edu

Received May $20^{\text {th }}, 2013$; revised June $21^{\text {st }}, 2013$; accepted July $5^{\text {th }}, 2013$

Copyright (C) 2013 Joseph Marino et al. This is an open access article distributed under the Creative Commons Attribution License, which permits unrestricted use, distribution, and reproduction in any medium, provided the original work is properly cited.

\begin{abstract}
Background and Objectives: Based on the case reports of hemorrhagic complications, recommendations for the removal of lumbar plexus catheters in anticoagulated patients were created. These guidelines are controversial as they limit the use of lumbar plexus blocks in postoperative anticoagulated patients. This study was designed to evaluate the incidence of hemorrhagic complications and coagulation status using International Normalized Ratio (INR) at the time of lumbar plexus catheter removal in patients receiving warfarin after total hip replacement. Methods: A retrospective study of 371 patients on warfarin thromboprophylaxis who received continuous lumbar plexus catheters for postoperative analgesia after total hip surgery was performed. The primary outcome measure was the incidence of bleeding complications after catheter removal; secondary outcome measures included warfarin dose, bridge therapy, incidence of deep vein thrombosis, pulmonary embolism (DVT/PE) and INR values upon catheter removal. Results: Almost all lumbar plexus catheters $(93 \% ; 344 / 371)$ were removed at 72 hours. At the time of catheter removal, mean INR was 1.99 [1.42 - 2.41] $(p=0.015) ; 67 \%$ of patients had an INR > 1.5 and half of these patients had INRs between $2.0-3.0 ; 5 \%$ had INR's between 3.0 - 4.0. There were no adverse bleeding complications or nerve injury after the removal of catheters. Conclusions: We observed no incidence of bleeding after lumbar plexus catheter removal despite $67 \%$ of patients demonstrating INR's $>1.5$. Our retrospective analysis illustrates the relative safety of catheter removal in anticoagulated patients and suggests that the removal of lumbar plexus catheters can be safely performed with an INR $>1.5$ in patients receiving warfarin.
\end{abstract}

Keywords: Lumbar Plexus Block; Warfarin; Prophylaxis; Deep Vein Thrombosis; Pulmonary Embolism

\section{Introduction}

Deep venous thrombosis and pulmonary embolism (DVT/ PE) prophylaxis guidelines call for the use of adjustedwarfarin or low molecular weight heparin in total hip arthroplasty patients [1-3]. Regional anesthesia practice guidelines in these anticoagulated patients previously addressed the question of the safety of neuraxial techniques following reports of increased risk of spinal he- matoma, a rare but serious complication [4]. These recommendations, to some degree, prompted a resurgence of peripheral nerve block techniques with lumbar plexus block, an effective analgesic technique in total hip arthroplasty patients $[5,6]$.

In 2010 revised recommendations were issued in response to case reports of severe hemorrhagic complications in anticoagulated patients undergoing deep plexus or peripheral blocks [7-11]. These recommendations pro- 
posed that while probably "more restrictive than necessary," practice guidelines originally developed specifically for neuraxial blockade techniques should also be applied to deep plexus and peripheral blockade techniques. These recommendations are controversial not only given the lack of available clinical study data but their divergence with therapeutic anticoagulation recommendations and their implications for post operative pain management in patients undergoing major orthopedic surgery.

Warfarin dosing is evaluated using the International Normalized Ratio (INR). Therapeutic anticoagulation recommendations for patients undergoing hip arthroplasty are to maintain INRs at $2.5(2.0-3.0)$. Current regional anesthesia guidelines, call for catheter removal while the INR is less than 1.5 [7]. The resultant early removal of lumbar plexus catheters may deprive patients of superior analgesia, adversely affecting their postoperative recovery and functional rehabilitation.

There is limited data available concerning the safety of peripheral blocks in anticoagulated patients. In 2006, Buckenmaier et al. reported no continuous peripheral nerve block related bleeding complications in a retrospective study of combat casualties receiving low-molecular weight heparin [12]. More relevant to our study population, Chelly et al. reported on the safe removal of lumbar plexus block catheters in total hip replacement patients anticoagulated with warfarin for 2 days following surgery. However, only $11 \%$ of patients from this study had a therapeutic INR value between 2 and 3 on the day of removal [13]. Evidently additional studies are required to satisfy the hypothesis that the safe removal of deep and/or plexus catheter is possible in therapeutically anticoagulated patients following hip replacement surgery.

The primary aim of this study was to assess retrospectively the incidence of hemorrhagic complications in therapeutically anti-coagulated patients upon removal of lumbar plexus blocks 3 days postoperatively after the initiation of warfarin for DVT prophylaxis.

\section{Methods}

The Institutional Review Board of Huntington Hospital approved this retrospective study and the data of 371 patients was reviewed over the period of June 2003-November 2010. Patients undergoing total hip replacement, receiving warfarin and continuous lumbar plexus nerve block for postoperative analgesia at Huntington hospital were included in the study. Patients were operated on by 11 different orthopedic surgeons, each of whom followed a clinical pathway which included the use of warfarin as the primary anticoagulant for therapeutic thromboprophylaxis.

\subsection{Study Design}

The continuous lumbar plexus block was performed preoperatively prior to the placement of the spinal anesthetic by one of two anesthesiologists (JM, JC) with an $18 \mathrm{G}$ insulated Tuohy needle connected to a nerve stimulator (Stimuplex Dig; B. Braun Medical, Bethlehem, Pennsylvania). The optimal needle placement was verified by a brisk quadriceps contraction (proximal movement of the patella) with a current output of $0.5 \mathrm{~mA}$, frequency of 2 $\mathrm{Hz}$, and pulse width of $100 \mu \mathrm{s}$. Patients received a bolus of $0.6 \mathrm{~mL} / \mathrm{kg}$ of $0.5 \%$ ropivacaine in slow fractionated aliquots through the perineural catheter after it was advanced $3 \mathrm{~cm}$. past the introducing needle. The position of the lumbar plexus catheter was confirmed with an injection of $10 \mathrm{~mL}$ of iohexol contrast medium (Omnipaque 180; Amersham Health, Oslo, Norway), followed by an anteroposterior radiograph as shown in Figure 1. Prior to discharge from the post anesthetic care unit, the perineural catheter was connected to an infusion pump (Abbott Plum A+; Abbott Laboratories, Illinois, USA) set up to infuse $0.2 \%$ ropivacaine at a rate of $0.15 \mathrm{~mL} / \mathrm{kg} / \mathrm{hr}$. for 48 hours. Warfarin prophylaxis was administered postoperatively on the day of surgery. Dosage was $2.5,5.0$, 7.5 or $10 \mathrm{mg}$ and was determined based on patient weight. Depending on the surgeon's preference, patients were placed on a bridge medication consisting of enoxaparin, heparin, dalteparin or clopidrogel until a therapeutic INR (2.0 - 3.0) level was achieved.

The removal of each lumbar plexus catheter was scheduled on postoperative day 3 , without any consideration of the INR value. Removal was performed by the nurse assigned to the care of the patient. After the removal of the perineural catheter, the nurse was instructed to monitor the patient for clinical evidence of perineural hematoma (pain at the site, morphologic changes) and neurologic deficits. Serial hematocrit levels were also followed by the medical staff and any acute decrease was followed with a diagnostic CT scan. Incidence of DVT was documented with the assistance of lower extremity

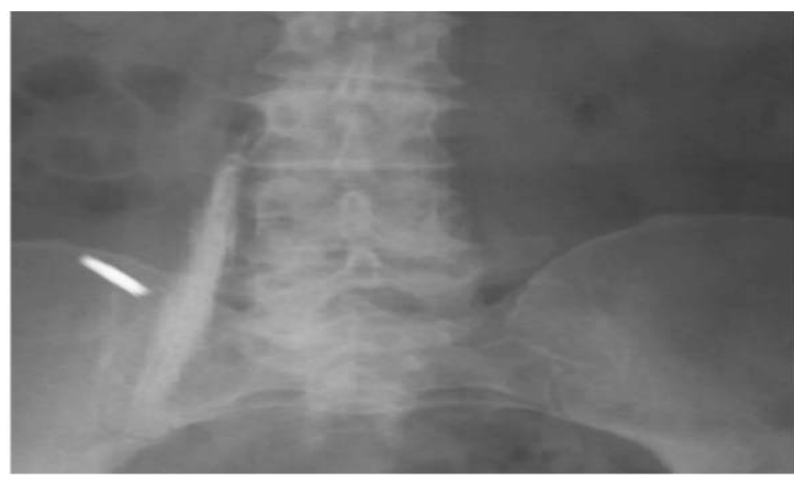

Figure 1. Radiographic view of iohexol spread with psoas muscle confirming optimal placement of catheter tip. 
Doppler ultrasound exams; patients with a suspected PE underwent a diagnostic $\mathrm{CT}$ scan.

\subsection{Statistical Analysis}

The distribution of INR in patients receiving thromboprophylaxis after a total hip replacement at the time of the lumbar plexus catheter removal is presented according to the thromboprophylaxis guidelines [1,4]: $0.9-1.4$ (normal), 1.5 - 1.9 (subtherapeutic), 2.0 - 3.0 (therapeutic), and $>3.1$ (over the therapeutic range). The incidence of major bleeding or DVT/PE upon removal of the lumbar plexus catheter was estimated using standard methods of estimating proportions along with their corresponding exact binomial confidence intervals. Descriptive statistics such as means, medians, standard deviations and range were also calculated. Comparisons between demographic variables such as age, weight, height and INR values upon removal of catheters were carried out using the Mann-Whitney test.

\section{Results}

\subsection{Patients}

Three hundred and seventy one postoperative primary hip arthroplasty patients anticoagulated with warfarin were included in this retrospective study. Patient characteristics, including demographics, body mass index (BMI), postoperative INR values at 72 hours post-op, are presented in Table 1.

\subsection{Incidence of Bleeding Complications after Catheter Removal}

The postoperative day of removal of lumbar plexus catheters is shown in Figure 2. Almost all (93\%; 344/ 371) catheters were removed on postoperative day 3. Twenty two catheters were inadvertently pulled out on postoperative day 2 ; one catheter was inadvertently removed on postoperative day 1 . Four catheters were removed after postoperative day 3 . There were no reported bleeding complications in any patients irrespective a mean INR of 1.99 on postoperative day 3 .

\subsection{Warfarin Dose and INR Value upon Removal of Catheters}

The median (range) dose of warfarin was $5 \mathrm{mg}(0-10)$ on the day of surgery, $5 \mathrm{mg}(0-10)$ on postoperative days 1 and 2 , and $2.5 \mathrm{mg}(0-10)$ on postoperative day 3 , the day of lumbar plexus catheter removal.

The distribution of warfarin dose on the day of surgery, postoperative day 1 and 2 and the day of removal of the lumbar plexus catheter are presented in Table 2. The INR values for study patients at the time of the lumbar
Table 1. Patient characteristics.

\begin{tabular}{ccccccc}
\hline & $\begin{array}{c}\text { Total } \\
n=371\end{array}$ & $\begin{array}{c}\text { Male } \\
n=135\end{array}$ & $\begin{array}{c}\text { Female } \\
n=236\end{array}$ & $\begin{array}{c}\text { Lower } \\
\text { Quartile }\end{array}$ & $\begin{array}{c}\text { Upper } \\
\text { Quartile }\end{array}$ & $p$-Value \\
\hline Age (years) & $\begin{array}{c}68.71 \\
\pm 12.07\end{array}$ & $\begin{array}{c}65.68 \\
\pm 12.61\end{array}$ & $\begin{array}{c}70.48 \\
\pm 11.40\end{array}$ & 60.29 & 77.04 & $<0.001$ \\
& 167.71 & 176.73 & 162.51 & & & \\
Height (cm) & \pm 10.29 & \pm 7.89 & \pm 7.57 & 160.02 & 175.26 & $<0.001$ \\
& 82.35 & 94.27 & 75.51 & & & \\
Weight (kg) & \pm 20.43 & \pm 16.89 & \pm 19.13 & 68.00 & 95.00 & $<0.001$ \\
& 29.13 & 30.13 & 28.56 & & & \\
BMI & $\begin{array}{c}10.02 \\
\left(\mathrm{~kg} / \mathrm{m}^{2}\right)\end{array}$ & \pm 6.65 & \pm 6.62 & 25.14 & 31.63 & 0.00030 \\
INR@72hrs & 1.99 & 1.85 & 2.07 & & & \\
post-op & \pm 0.74 & \pm 0.64 & \pm 0.78 & 1.42 & 2.41 & 0.015 \\
\hline
\end{tabular}

Data are presented as mean $\pm \mathrm{SD}$.

Table 2. Distribution of warfarin dosing.

\begin{tabular}{ccccc}
\hline $\begin{array}{c}\text { Warfarin } \\
\text { Dosage }\end{array}$ & $\begin{array}{c}{ }^{1} \text { DOS } \\
(n=371)\end{array}$ & $\begin{array}{c}{ }^{2} \text { POP Day 1 } \\
(n=371)\end{array}$ & $\begin{array}{c}\text { POP Day 2 } \\
(n=371)\end{array}$ & $\begin{array}{c}\text { POP Day 3 } \\
(n=371)\end{array}$ \\
\hline $2.5 \mathrm{mg}$ & $14(3.78)$ & $24(6.49)$ & $71(19.19)$ & $193(51.89)$ \\
$5 \mathrm{mg}$ & $202(54.32)$ & $341(91.89)$ & $285(76.76)$ & $151(40.81)$ \\
$7.5 \mathrm{mg}$ & $85(22.97)$ & $6(1.62)$ & $14(3.78)$ & $21(5.68)$ \\
$10 \mathrm{mg}$ & $70(18.92)$ & - & $1(0.27)$ & $6(1.62)$ \\
\hline
\end{tabular}

${ }^{1}$ Day of surgery; ${ }^{2}$ Postoperative; As none of the patients developed bleeding complications or deep vein thrombosis (DVT) the proportion of patients without bleeding or DVT was $100 \%$ (exact $95 \%$ CI; $(99 \%, 100 \%$ ).

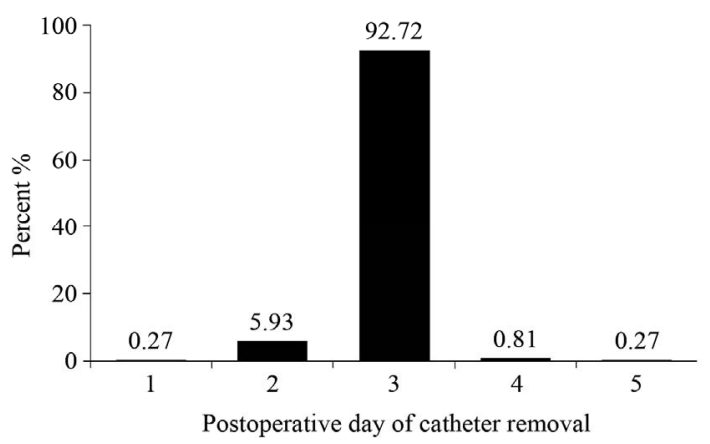

Figure 2. Day of lumbar plexus block catheter removal.

plexus catheter removal are presented in Figure 3. The mean INR was $1.99[1.42-2.41](p=0.015)$ on postoperative day 3 upon lumbar plexus catheter removal. Sixty-seven percent of patients had an INR greater than 1.5 on postoperative day 3 . Almost $25 \%$ of patients had an INR between 2.0 and 3.0 at the time of catheter removal, while $12 \%$ of patients had INR's greater than 3.0.

\subsection{Bridge Therapy and Incidence of DVT/PE}

Ninety-three percent of our patients were "bridged" with a different thromboprophylactic medication while waiting for therapeutic levels to be achieved with warfarin. The majority of patients were placed on enoxaparin $(52.97 \%)$, followed by heparin $(28.65 \%)$ then dalteparin 


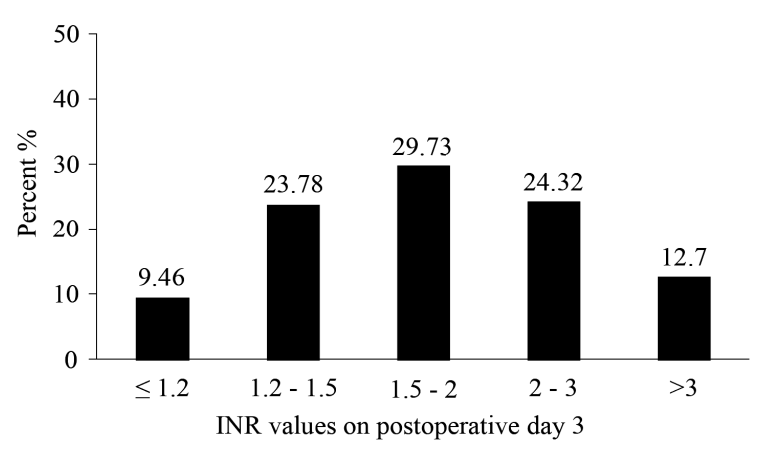

Figure 3. INR values on postoperative day 3 .

(10.54\%). Only one patient was placed on clopidrogel. Seven percent of patients received no bridging medication. There were no reported bleeding complications, $\mathrm{DVT} / \mathrm{PE}$ or incidences of nerve injury in any of the patients.

\section{Discussion}

Patient coagulation status at the time of removal of deep perineural catheters is a controversial anesthetic practice question. Achieving therapeutic warfarin anticoagulation with an INR range of 2.0 - 3.0 to prevent DVT and PE is inconsistent with current recommendations for the removal of lumbar plexus blocks at INR values of less than 1.5 [7]. This discrepancy in guidelines, especially given the lack of clinical study data, underlines the need for studies demonstrating a balanced approach to safe, effective postoperative pain management and therapeutic anticoagulation.

In this retrospective analysis of data from charts of 371 patients, we observed no adverse bleeding complications upon removal of lumbar plexus catheters 3 days after initiation of warfarin thromboprophylaxis. At the time of catheter removal, over half of our patients $(67 \%)$ had INRs greater than 1.5, more than a third of these patients had therapeutic INRs of greater than 2.0. Forty seven of these patients had INR values greater than 3 .

The safe removal of lumbar plexus catheters in patients anti-coagulated with warfarin is supported by reports that INR values in the first 3 days of warfarin therapy may not accurately reflect adequate thrombolytic activity. Although INR values are elevated, Factor VII levels may still be sufficient for hemostasis, a theory also proposed by Liu et al when reporting on the safe removal of epidural catheters in patients with INRs of greater than 1.4 during the initiation of warfarin therapy $[14,15]$. This inconsistency between INR levels and thrombolytic activity may provide further protection from bleeding complications during the removal of lumbar plexus catheters from the vascular psoas muscle as real-time therapeutic anticoagulation is not achieved.

Our findings are consistent with other retrospective studies in similar patient populations. Chelly and Schilling reported on the safe removal of perineural catheters in joint replacement patients on a variety of thromboprophylactic regimens including warfarin [16]. In a similar study model, Chelly et al. affirmed the safe removal of lumbar plexus catheters in hip replacement patients with INR's after the second postoperative day [13]. While our study and others like it suffer many of the limitations of a retrospective review, they still demonstrate that anesthesiologists can, with appropriate assessment of the individual patient's risks of thromboembolism and bleeding, utilize regional anesthesia techniques in postoperative pain management of anti-coagulated patients. Also worth considering in this patient risk assessment is the clinical setting and the level of skill required in performing this type of peripheral block.

Also noteworthy in our patient cohort was the lack of prevalence of DVT. In the United States, the incidence of DVT in postoperative hip replacement patients has been reported to range from $12 \%-23 \%$, even when using anticoagulant prophylaxis [17]. Our data demonstrated that only $37 \%$ of patients achieved the recommended therapeutic INR 3 days after surgery at the time of lumbar plexus catheter removal and $7 \%$ of patients were not bridged with any medication. Early mobilization due to improved analgesia and decreased opioid-related side effects may, in part, explain the lack of DVT in our patient cohort.

\section{Conclusion}

Our findings demonstrate that the uncomplicated removal of lumbar plexus catheters from postoperative anti-coagulated hip replacement patients is possible with INR values of greater than 1.4. Our results, when taken in the context of other retrospective studies illustrate the likelihood that current recommendations for the maintenance of lumbar plexus catheters in anti-coagulated patients may be too conservative leading to their premature removal, often depriving patients of effective postoperative analgesia. In addition to the lack of bleeding complications, effective postoperative analgesia translates to early mobilization, which may be associated with the decreased incidence of DVT. These data suggest that, with appropriate consideration of individual risk factors for bleeding and thromboembolism, patients anti-coagulated with warfarin could benefit safely from the superior postoperative analgesic effects of continuous lumbar plexus blocks, leading to the early mobilization required to reduce postoperative complications.

\section{REFERENCES}

[1] B. N. Stulberg, J. N. Insall, G. W. Williams and B. Gel- 
man, "Deep-Vein Thrombosis Following Total Knee Replacement. An Analysis of Six Hundred and Thirty-Eight Arthroplasties," The Journal of Bone and Joint Surgery, Vol. 66, No. 2, 1984, pp. 194-201.

[2] A. N. Nicolaides, H. K. Breddin, J. Fareed, S. Goldhaber, S. Haas, R. Hull, et al., "Prevention of Venous Thromboembolism. International Consensus Statement. Guidelines Compiled in Accordance with the Scientific Evidence," International Angiology, Vol. 20, No.1, 2001, pp. 1-37. doi:10.1177/000331970105200101

[3] W. H. Geerts, D. Bergqvist, G. F. Pineo, J. A. Heit, C. M. Samama, M. R. Lassen, et al., "Prevention of Venous Thromboembolism: American College of Chest Physicians Evidence-Based Clinical Practice Guidelines (8th edition)," Chest, Vol. 133, No. 6, 2008, pp. 133,381S$453 \mathrm{~S}$.

[4] T. T. Horlocker, D. J. Wedel, H. Benzon, D. L. Brown, F. K. Enneking, J. A. Heit, et al., "Regional Anesthesia in the Anticoagulated Patient: Defining the Risks (the Second ASRA Consensus Conference on Neuraxial Anesthesia and Anticoagulation," Regional Anesthesia and Pain Medicine, Vol. 28, No. 3, 2003, pp. 172-197.

[5] C. C. Buckenmaier 3rd and L. L. Bleckner, "Continuous Peripheral Nerve Blocks and Anticoagulation," British Journal of Anaesthesia, Vol. 101, No. 2, 2008, pp. 139140. doi:10.1093/bja/aen192

[6] J. Marino, J. Russo, M. Kenny, R. Herenstein, E. Livote and J. E. Chelly, "Continuous Lumbar Plexus Block for Postoperative Pain Control after Total Hip Arthroplasty. A Randomized Controlled Trial," The Journal of Bone and Joint Surgery, Vol. 91, No. 1, 2009, pp. 29-37.

[7] T. T. Horlocker, D. J. Wedel, J. C. Rowlingson, F. K. Enneking, S. L. Kopp, H. T. Benzon, et al., "Regional Anesthesia in the Patient Receiving Antithrombotic or Thrombolytic Therapy: American Society of Regional Anesthesia and Pain Medicine Evidence-Based Guidelines (Third Edition)," Regional Anesthesia and Pain Medicine, Vol. 35, No. 1, 2010, pp. 64-101.

[8] S. Aida, H. Takahashi and K. Shimoji, "Renal Subcapsular Hematoma after Lumbar Plexus Block," Anesthesiology, Vol. 84, No. 2, 1996, pp. 452-455. doi:10.1097/00000542-199602000-00027

[9] C. Aveline and F. Bonnet, "Delayed Retroperitoneal Haematoma after Failed Lumbar Plexus Block," British Journal of Anaesthesia, Vol. 93, No. 93, 2004, pp. 589-591.

\section{doi:10.1093/bja/aeh242}

[10] S. M. Klein, F. D’Ercole, R. A. Greengrass and D. S. Warner, "Enoxaparin Associated with Psoas Hematoma and Lumbar Plexopathy after Lumbar Plexus Block," Anesthesiology, Vol. 87, No. 6, 1997, pp. 1576-1579. doi:10.1097/00000542-199712000-00040

[11] P. Bickler, J. Brandes, M. Lee, K. Bozic, B. Chesbro and J. Claassen, "Bleeding Complications from Femoral and Sciatic Nerve Catheters in Patients Receiving Low Molecular Weight Heparin," Journal of Anesthesia and Analgesia, Vol. 103, No. 4, 2006, pp. 1036-1037. doi:10.1213/01.ane.0000237230.40246.44

[12] C. C. Buckenmaier 3rd, C. H. Shields, A. A. Auton, S. L. Evans, S. M. Croll, L. L. Bleckner, et al., "Continuous Peripheral Nerve Block in Combat Casualties Receiving Low-Molecular Weight Heparin," British Journal of Anaesthesia, Vol. 97, No. 6, 2006, pp. 874-877. doi:10.1093/bja/ael269

[13] J. E. Chelly, D. M. Szczodry and K. J. Neumann, "International Normalized Ratio and Prothrombin Time Values before the Removal of a Lumbar Plexus Catheter in Patients Receiving Warfarin after Total Hip Replacement," British Journal of Anaesthesia, Vol. 101, No. 2, 2008, pp. 250-254. doi:10.1093/bja/aen132

[14] H. T. Benzon, M. J. Avram, H. A. Benzon, M. KirbyNolan and A. Nader, "Factor VII Levels and International Normalized Ratios in the Early Phase of Warfarin Therapy," Anesthesiology, Vol. 112, No. 2, 2010, pp. 298304. doi:10.1097/ALN.0b013e3181ca6cfc

[15] S. S. Liu, A. Buvanendran, E. R. Viscusi, E. Hutton, T. Lubenow, J. Zhou, et al., "Uncomplicated Removal of Epidural Catheters in 4365 Patients with International Normalized Ratio Greater than 1.4 during Initiation of Warfarin Therapy," Regional Anesthesia and Pain Medicine, Vol. 36, No. 3, 2011, pp. 231-235. doi:10.1097/AAP.0b013e31820d4376

[16] J. E. Chelly and D. Schilling, "Thromboprophylaxis and Peripheral Nerve Blocks in Patients Undergoing Joint Arthroplasty," The Journal of Arthroplasty, Vol. 23, No. 3, 2008, pp. 350-354. doi:10.1016/j.arth.2007.05.045

[17] J. R. Lieberman and W. H. Geerts, "Prevention of Venous Thromboembolism after Total Hip and Knee Arthroplasty," The Journal of Bone and Joint Surgery, Vol. 76, No. 8, 1994, pp. 1239-1250. 